\title{
Impact of the COVID-19 pandemic on human papillomavirus-based testing services to support cervical cancer screening
}

\author{
Mario Poljak ${ }^{1}$, Kate Cuschieri², Dur-e-Nayab Waheed ${ }^{3}$, Marc Baay ${ }^{4}$ Alex Vorsters $^{3}$ M $^{\infty}$
}

${ }^{1}$ Institute of Microbiology and Immunology, Faculty of Medicine, University of Ljubljana, Ljubljana, Slovenia. ${ }^{2}$ Scottish HPV Reference Laboratory, Royal Infirmary of Edinburgh, NHS Lothian Scotland, Edinburgh, United Kingdom. ${ }^{3}$ Centre for Evaluation of Vaccination, Vaccine and Infectious Diseases Institute, University of Antwerp, Wilrijk, Belgium. ${ }^{4} 995$ Epidemiology \& Pharmacovigilance, Leuven, Belgium.

\begin{abstract}
Introduction: The World Health Organization elimination goal for cervical cancer relies on screening $70 \%$ of women at ages 35 and 45, preferentially through molecular HPV testing. The SARS-CoV-2 pandemic has led to an unprecedented demand for molecular tests and platforms. Our objective was to gain insight into the impact of SARS-CoV-2 on the actual or anticipated shortage of tests, equipment, consumables, and staff required to deliver molecular HPV laboratory services and to consider the implications for the sustainability and development of cervical screening programs.

Methods: A 19-item online questionnaire was created and made available online between December 2020 and February 2021. Five companies with clinically validated HPV and SARS-CoV-2 tests in their portfolios were invited to provide a statement on the volumes of molecular COVID-19 tests produced, relevant changes to manufacturing capacity, and their current and post-pandemic strategy for HPV tests.

Results: We received responses from 57 laboratories representing 30 countries and six continents. Among these, $74 \%$ reported experiencing a supply shortage, $54 \%$ reported a shortage of personnel, and $33 \%$ reported delays in ordering equipment. Three companies described expansion of manufacturing lines, investment in diagnostic infrastructure, and scale-up of manufacturing capacity. Two companies specifically referred to opportunities for the use of platforms for COVID-19 testing to support HPV testing in time.

Conclusions: The demand for SARS-CoV-2 testing is competing with HPV testing, compounded by a shortage of staff. This represents a challenge for existing laboratory services and for settings keen to implement HPV-based screening. However, supply challenges may be addressed in time, given the significant investment in manufacturing capacity. In addition, innovation around molecular COVID-19 testing systems may result in solutions that address the shortage of rapid low-cost HPV testing systems for low-resource settings. Finally, because the demand for COVID-19 testing is likely to decrease, this may release both workforce and platform capacity for high-throughput HPV testing. The global health community should be alert to the opportunities around innovation and capacity if cervical cancer elimination goals are to be reached.
\end{abstract}

Keywords: molecular testing, COVID-19, human papillomavirus, HPV, cervical cancer screening

Received: 2 March 2021 | Returned for modification: 3 March 2021| Accepted: 7 March 2021

\section{Introduction}

In 2018, the World Health Organization's (WHO) director-general called for action to eliminate cervical cancer, with elimination defined as an incidence of less than four cases per 100,000 (1). Three key pillars are required and available to reach this goal: primary prevention through human papillomavirus (HPV) vaccination, ideally before onset of sexual activity; secondary prevention through screening, with relevant follow-up of screen-positive women; and, finally, timely treatment of precancerous lesions and invasive cancer. Although the goal of elimination could ultimately be reached with intensive HPV vaccination alone, it can be achieved earlier by a combination of pragmatic but effective screening and vaccination. Therefore, the targets are to fully vaccinate $90 \%$ of girls before age 15 , to screen $70 \%$ of women with a high-performance test at ages 35 and 45, and to provide treatment and care to $90 \%$ of women with a diagnosed cervical disease. If $95 \%$ of countries manage to rapidly adopt the 90-70-90 targets, the goal of cervical cancer elimination may become a reality as early as $2030(2,3)$.

These goals are ambitious, and optimal cervical cancer screening demands large-scale testing using molecular HPV tests. In a recent survey, 254 distinct HPV tests were identified on the global market (4). Unfortunately, $60 \%$ of these tests are without a single peer-reviewed publication, and over $90 \%$ are not evaluated in line with accepted international performance metrics that ensure accuracy in screening settings (4). To promote and facilitate access to safe, reliable, and appropriate in vitro diagnostic technologies, the WHO performs prequalification of in vitro diagnostics for high-burden diseases. Currently, only three tests for HPV-based screening have been WHO prequalified (5). To summarize, there is still a relatively small pool of HPV tests that fulfill the operational and performance characteristics required to meet the global screening challenge.

Clearly the COVID-19 pandemic has had a major impact on global health. As of March 2021, nearly 115 million people have been infected with SARS-CoV-2, and more than 2.6 million have died from COVID-19 (6). The restrictions (e.g., social distancing, lockdown, and suspension of non-essential clinical services) imposed to reduce the spread of SARS-CoV-2 have led to reductions in the overall number of cancers diagnosed, with an increased proportion of cancer diagnoses at advanced stages. According to a study conducted by the WHO, 54\% of countries reported disrupted cancer-treatment services during the first COVID-19 pandemic 
wave in spring 2020. The closure of population-level screening programs was among the top five most common disruptions to services across non-communicable diseases noted by the WHO (7). Data from the nationwide Netherlands Cancer Registry for the period between February 24th and April 12th, 2020 showed that there was a notable decrease in overall cancer diagnoses when compared to the pre-pandemic period (8). Similar data have been reported for Austria, Denmark, Germany, Poland, the United Kingdom, and the United States (9-14).

The HPV Prevention and Control Board (http://www.hpvboard. org) is an independent international multidisciplinary group of experts that provide evidence-based reflection and guidance on strategic, technical, and policy issues that will occur as we move forward in the implementation of HPV control and prevention programs. The board assists in monitoring and evaluating national and regional control programs. In August 2020, the board held a meeting to discuss challenges in the HPV-based cervical cancer screening landscape, which included a session on the impact of COVID-19 on screening programs (15). The discussion in that particular session prompted the board to hold an additional 2-day meeting in November 2020 fully devoted to the impact of COVID-19 on cervical cancer screening and treatment, and HPV vaccination (16). At this meeting, the board members noted that COVID-19 had not only impacted screening attendance, but the availability and supply of consumables, equipment, and staff required for HPVbased screening. Given that these observations were based on the experience of those in the board, either directly and/or through anecdotal exchange(s) with colleagues, it was agreed that this important issue may be better understood through the creation and wide dissemination of a brief survey.

The purpose of the survey was to acquire real-life insight into the impact of SARS-CoV-2 on potential shortages of tests, equipment, consumables, and staff required to effectively run HPV-specific laboratory procedures. The survey also served as an opportunity to determine common challenges and to identify potential opportunities that may support sustainable HPV-based cervical cancer screening in the future.

We also contacted five major manufacturers of clinically validated HPV tests to gain an understanding of their plans to mitigate current and future pressures on the demand for molecular testing.

\section{Methods}

An online questionnaire was developed using Qualtrics ${ }^{\mathrm{XM}}$ (London, UK) to investigate the impact of the COVID-19 pandemic on HPV-based cervical cancer screening in reference and screening laboratories worldwide. The questionnaire contained 19 items, mostly multiple choice, but with room to provide additional freetext comments. The questionnaire was tested for comprehensibility and clarity in a small pilot. The full questionnaire is available at https://www.uantwerpen.be/en/projects/hpv-prevention-andcontrol-board/resources/.

The survey was circulated among International Papillomavirus Society (IPVS) members and laboratories participating in the Global HPV LabNet DNA Genotyping Proficiency Panels in 2018 and 2020 (which serves as an External Quality Assurance Scheme), and it was included in the European Society for Clinical Microbiology and Infectious Diseases (ESCMID) member newsletter. The questionnaire targeted laboratory managers with responses limited to one per laboratory. The questionnaire was available online between December 21st, 2020 and February 2nd, 2021.
IBM SPSS Statistics 27 was used to perform the data analysis, and Excel (Microsoft, Redmond, WA, USA) was used for data presentation. Only descriptive analyses were performed.

To contextualize the responses of users with those from industry, a limited number of companies with a molecular portfolio that includes clinically validated HPV and SARS-CoV-2 test(s) (Roche Diagnostics, Indianapolis, IN, USA; Abbott Laboratories, Chicago, IL, USA; Cepheid, Sunnyvale, CA, USA; Becton Dickinson, Franklin Lakes, NJ, USA; and Hologic, Marlborough, MA, USA) were invited to provide a statement on support for the emerging need by national and regional governments for high volumes of molecular COVID-19 testing; recent investment in manufacturing capacity, diagnostic infrastructure, and the supply chain; safeguards and commitment to manufacture other molecular diagnostic assays (including HPV tests); and, finally, their current and post-pandemic strategy for HPV tests.

\section{Results}

We received responses from 57 laboratories, from 30 countries across six continents, representing 19 high-income countries and 11 low- and middle-income countries (Fig. 1). Eight responses were excluded from the analysis because of incomplete data. The only information that could be retrieved from the excluded responses was the name, location, and nature of the laboratory, and how HPV testing was applied. Three additional responses were from laboratories that did not perform HPV tests, which were also excluded. The 46 laboratories, all routinely performing HPV testing, were distributed among general molecular diagnostic laboratories $(n=16)$, HPV reference laboratories $(n=13)$, combined general molecular and HPV reference laboratories $(n=8)$, and "other" $(n=9$; Table 1$)$.

Laboratories were asked whether they had experienced shortages of supplies for HPV testing such as sample collection/transport media, HPV tests, consumables, plasticware, and personal

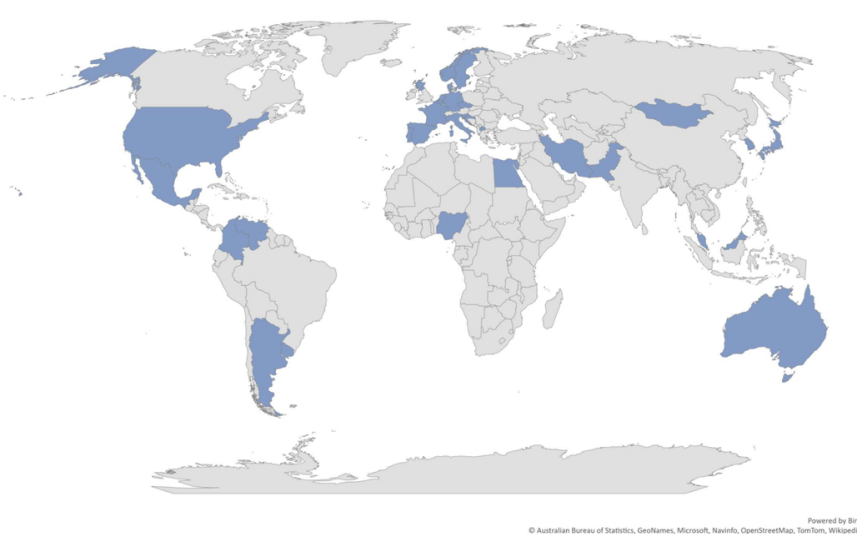

Figure 1 | Geographical distribution of survey respondents. Blue indicates countries that responded to the survey.

Table 1 | Types of laboratories that participated in the survey.

\begin{tabular}{lcc}
\hline Type of laboratory & $n$ & $\%$ \\
\hline General molecular diagnostic laboratory & 12 & 26 \\
HPV reference laboratory & 11 & 24 \\
Other & 9 & 20 \\
General molecular diagnostic laboratory and other & 4 & 9 \\
HPV reference laboratory and general molecular & 8 & 17 \\
diagnostic laboratory & & \\
HPV reference laboratory and other & 2 & 4 \\
\hline Total & 46 & 100 \\
\hline
\end{tabular}


protective equipment (PPE) during the past year. Out of 46 responses, 34 laboratories (73.9\%) reported a supply shortage of at least one of the items listed (Table 2). Shortages were reported of the following, in decreasing order: DNA/RNA extraction reagents and consumables, plasticware, polymerase chain reaction (PCR) reagents, and sample collection and transport media.

With respect to personnel during the pandemic, 25 laboratories $(54.3 \%)$ reported experiencing a shortage. Frequently listed reasons for this were, in decreasing order: staff co-opted to support COVID-19 testing, limits on the number of staff that were able to work concurrently, and self-isolation (Table 3).

Next to shortage of supplies and staff, $32.6 \%$ of laboratories reported a delay in ordering equipment, and 19.6\% and $36.9 \%$ reported a higher purchase price for HPV-specific products or for non-HPV-specific products, respectively, in comparison to the prepandemic period.

Eighteen laboratories (39.1\%) reported that the time to HPV test results was significantly prolonged during COVID-19, with 12 of 18 laboratories indicating a delay of more than a month (Fig. 2). Similarly, 32 laboratories (69.5\%) anticipated that COVID-19

Table 2 | Shortage of specific consumables during COVID-19 pandemic reported by respondents.

\begin{tabular}{lc}
\hline Supply shortage & $\begin{array}{c}\text { Number of times } \\
\text { ticked }\end{array}$ \\
\hline Collection and transport media & 11 \\
Plasticware: pipette tips, sample tubes, storage & 16 \\
boxes, etc. & 18 \\
Extraction reagents & 12 \\
PCR reagents & 4 \\
Swabs & 8 \\
Molecular-grade fluids (ethanol), general & 5 \\
disinfectants & 9 \\
Waste bins and waste collection services & 9 \\
Personal protective equipment &
\end{tabular}

\section{services}

Respondents could choose multiple options for this question and tick multiple consumables.

Table 3 | Reasons for shortage of personnel in the laboratory during COVID-19 pandemic reported by respondents.

\begin{tabular}{lc}
\hline Reasons for shortage of personnel & $\begin{array}{c}\text { Number of times } \\
\text { ticked }\end{array}$ \\
\hline Not all staff was allowed to work at the same time & 12 \\
Staff co-opted to support COVID-19 testing & 12 \\
Self-isolation & 6 \\
Other reasons & 3 \\
Not answered (no shortage) & 22 \\
\hline Respondents could choose multiple options for this question and tick multiple \\
reasons.
\end{tabular}

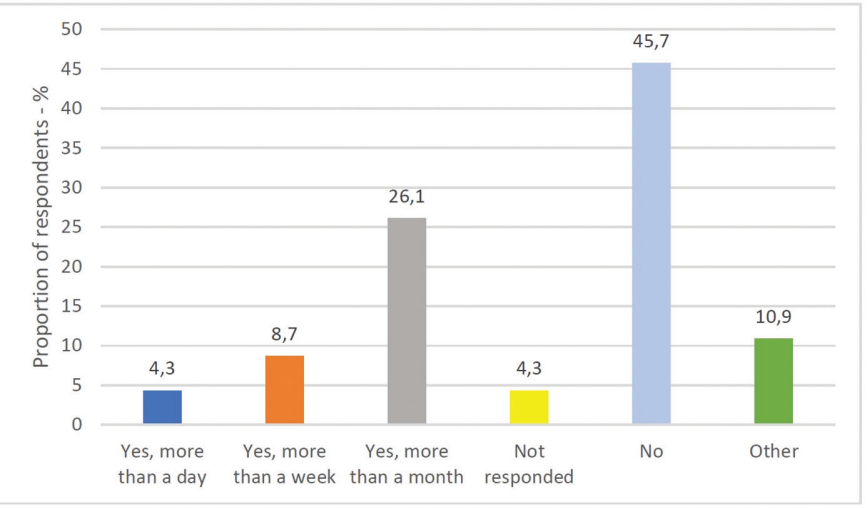

Figure 2 | Measure of frequency to determine if the time to receive HPV test results was prolonged during the COVID-19 pandemic. testing would impact HPV testing, either short-term (less than 6 months, 39.1\%) or long-term (at least 1 year, 30.4\%, Fig. 3). Twenty-nine laboratories $(63.0 \%)$ reported a temporary suspension of routine cervical cancer screening due to COVID-19 (Fig. 4). Finally, 25 laboratories (54.3\%) reported that COVID-19 had negatively impacted research and development capacity and activity (Fig. 5). Individual comments were provided by respondents on the impact of COVID-19 on HPV-based research. The option of selfsampling for HPV testing was frequently raised as a way to minimize face-to-face health care visits while increasing the number of women screened.

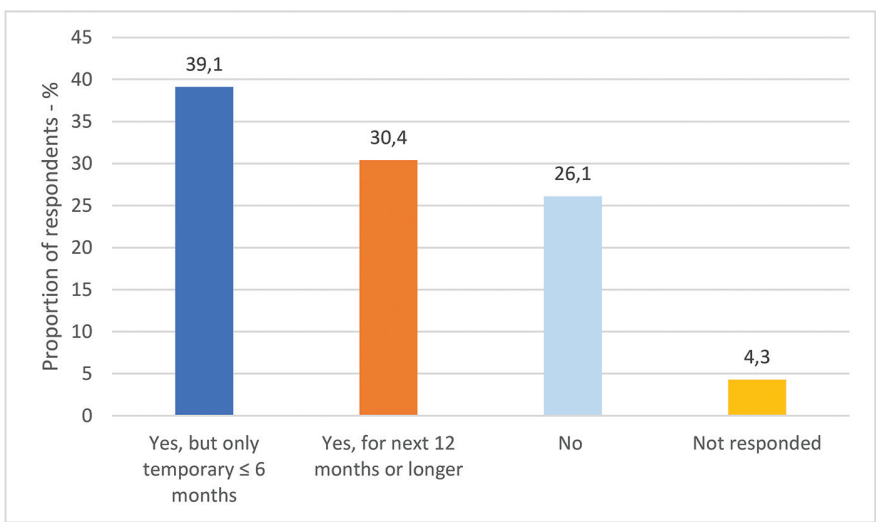

Figure 3 | Measure of frequency for impact of COVID-19 on HPV testing.

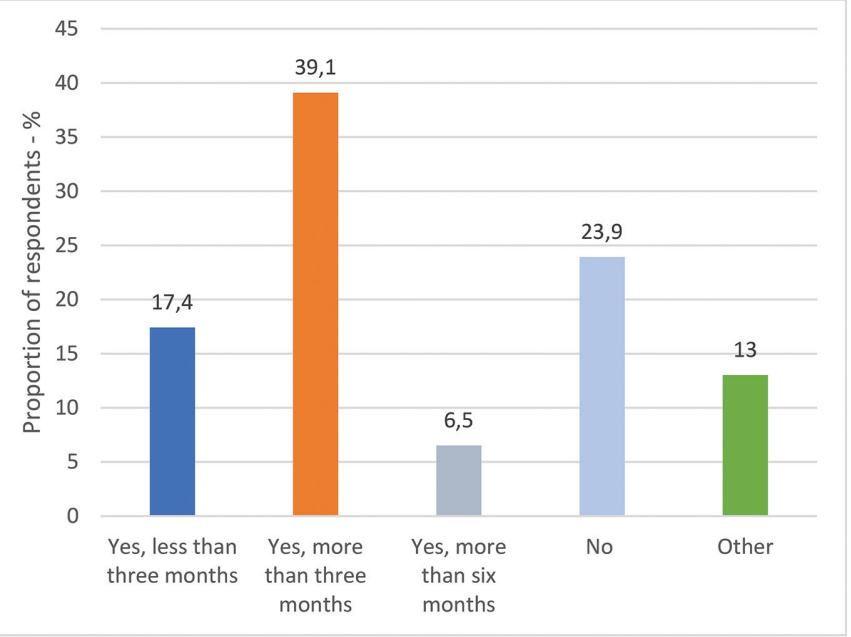

Figure 4 | Measure of frequency for suspension of routine cervical cancer screening program due to COVID-19.

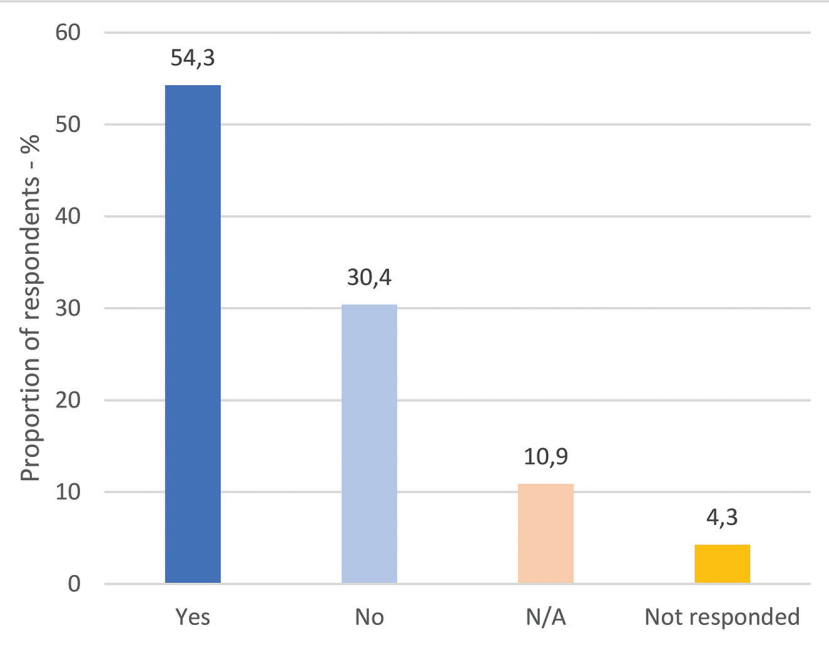

Figure 5 | Measure of frequency for impact of COVID-19 on research and development capacity and activity of the laboratory. N/A = not available. 
Finally, four companies provided a statement in response to a question about mitigation and plans to support and develop molecular microbiology testing during and after the COVID-19 era. All companies clearly expressed a commitment to being on the "front line" to support the emergency across the world for high volumes of COVID-19 testing. Consequently, all described substantial growth in diagnostics activity and business. Two companies described tremendous growth in sales of around $100 \%$, mainly driven by a continued unprecedented global demand for their portfolio of lab-based COVID-19 tests. In addition to sales, all companies described sizable increases in manufacturing capacity (including of emerging products) and investment in new supply chains and molecular and core laboratory solutions. To illustrate this point, one company reported that pre-pandemic they launched, on average, five microbiology assays per year, whereas in 2021 they anticipate a launch of 17 assays, including molecular, antigen, and serology assays. Commensurate with this was a reported doubling of PCR capacity in early 2021 and anticipated tripling of PCR capacity by the end of 2021. One company described a significant increase in manufacturing capacity; for example, adding 60 new manufacturing lines for consumables, with 17 new locations, and 20 new manufacturing lines for reagents, along with four major facility expansions as well as one new manufacturing space for instruments. It is anticipated that the increase in manufacturing capacity dispersed across the world should help create a regionally responsive supply of core reagents and consumables. Although responses about the near and distant future were understandably high-level, there was an acknowledgment that recent scale up and developments (and the structural "shifts" within the companies required to deliver these) are likely to have positive consequences for molecular diagnostics in general terms. Of interest, two companies specifically referred to opportunities for the use of platforms installed a priori for COVID-19 testing to support HPV testing in time. Two companies stated that with multimillion euro investment in manufacturing facilities they will safeguard the European supply not only for SARS-CoV-2 assays; this will also safeguard the manufacture of their other molecular diagnostic assays, including HPV assays.

\section{Discussion}

The points initially articulated at the 2020 HPV Prevention and Control Board meetings are consistent with the results of the survey presented here in that the majority of laboratories reported a demonstrable impact of the COVID-19 pandemic on day-to-day HPV testing activities and molecular testing in general. The more common issues involved shortages in consumables (particularly pipette tips, sample tubes, storage boxes, disinfectants, and PPE), kits, including extraction reagents and PCR reagents, and personnel, and to a lesser degree a delay in ordering equipment. Moreover, some laboratories reported a higher purchase price for both $\mathrm{HPV}$-specific and companion generic products.

In some settings, the survey identified longer turnaround times for receipt of HPV test results during the pandemic. Several factors, most probably synergistically, contributed to the delay: shortages in consumables and kits, and delays in supplies as well as various personnel-related problems: staff co-opted to COVID-19 testing, limitations on concurrent laboratory occupancy, self-isolation, and quarantine.

There are caveats and limitations to the survey and approach. Although the number of responses was somewhat limited, they represented a global sample, covering all continents, as shown in Figure 1. It is also possible that we received a disproportionate response from laboratories that had experienced issues or challenges, whereas laboratories that were unaffected may have felt less of an impulse to respond. Given the nature of the dissemination, we accept that the analysis is largely descriptive, but we nevertheless hope that it highlights and facilitates further discussion on what is an important issue. In addition, due to the study design, it was not possible to identify differences between the first and second pandemic waves concerning the impact of the COVID-19 pandemic on HPV-based testing.

Overall, the results suggest that the demand for SARS-CoV-2 testing is competing with the ability to deliver HPV testing, at least in the short term. This is compounded by issues with availability of trained staff, who may be either co-opted to work on COVID-19 or restricted in their working patterns due to their own illness or national or local infection-control measures and policies. Although no country reported a total breakdown in cervical screening service(s) due to issues of HPV kit supply or consumables, the situation points to an added vulnerability in service delivery and plans for development, which was unanticipated pre-pandemic, regardless of whether countries rely on HPV-based screening or use HPV tests for borderline cytology triage or test of cure. Furthermore, we may need to manage our expectations for how rapidly the increased demand for instrumentation, kits, and trained individuals required to address the cervical cancer elimination goals will be met.

It was also notable that laboratories described a decline in the ability to deliver HPV or other non-COVID-19-associated research and development as a consequence of the pandemic. Clearly, in service laboratories the enormous demands of the diagnostic repertoire take priority; however, the reduction in research and development capacity does not have trivial implications. It is often the service laboratories that have the skills, samples, and infrastructure to evaluate new HPV tests and diagnostic approaches rather than pure academic or university settings. Given that there was a critical need for more robust evaluation of commercial HPV technologies even pre-pandemic (4), this has consequences for the rapidity of evaluation, and thus implementation, of emerging tests.

However, in addition to the rather sobering issues above, we must also reflect on the potential and considerable silver linings. As indicated in the responses from the manufacturers, the expansion of existing molecular diagnostic portfolios and manufacturing capacity in recent times has had an unprecedented pace. General innovation around COVID-19-testing tools and recycling of neglected technologies, such as rapid testing systems based on low-cost portable instruments and low-complexity chemistry (e.g., microfluidic devices, various isothermal amplification technologies, or clustered regularly interspaced short palindromic repeats [CRISPR]-based technology) (17-19), may be converted in the near future to affordable screening assays for HPV, with applicability in low-resource settings (20). The validation of at least some of these new assays will be a major challenge to meet the worldwide need for HPV tests with acceptable performance characteristics required to respond to the cervical cancer elimination goal (21). The global HPV test supply needed to reach the $70 \%$ screening coverage aspiration of the WHO is estimated to be in the range of 1.4 to 1.5 billion HPV tests over a time span of 5 years (N. Broutet, personal communication).

In addition, although the laboratory workforce may currently be a limiting factor, given the issues described above, the speed of 
recruitment and/or cross-training of staff to support the organization and delivery of COVID-19 testing will ensure a future larger cohort of trained individuals (of various grades of seniority and experience) that will have skills that are highly transferable to general molecular systems, including HPV. In the same vein, fully integrated, high-throughput, automated sample-to-result molecular analyzers installed initially for COVID-19 testing are likely to experience redundancy in the future; this could be successfully occupied by large-scale HPV testing.

Finally, given that we may have to deal with COVID-19 for some time yet-and given the demands of the cervical cancer elimination goals-creative strategies that support increased and equitable access to screening become ever more pressing. Molecular HPV testing is amenable to self-taken samples. Self-sampling arguably should no longer be considered an option offered only to those considered "hard to reach"; the timing to explore this as a general screening approach seems more relevant than ever. Expanding the use of self-sampling should go hand in hand with increased production of swabs and sample collection and transport media because issues have already been encountered in sexually transmitted infection clinics.

In conclusion, the COVID-19 pandemic is likely to lead to a temporary delay in reaching the cervical cancer elimination targets, with the potential to catch up through repurposing of equipment, infrastructure, and human resources from COVID-19 testing. It is our shared responsibility to make sure that the targets are eventually reached, to remain alert to the new opportunities that have arisen from the COVID-19 pandemic, and to ensure they are smoothly implemented into the cervical cancer elimination strategy as well as in other fields of medicine.

\section{Acknowledgements}

The authors would like to thank the HPV Prevention and Control
Board advisors for their input in study design and discussion, all the laboratory managers that kindly completed the survey, the companies for their willingness to provide feedback, and the Global HPV LabNet, the International Papillomavirus Society (IPVS), and the European Society of Clinical Microbiology and Infectious Diseases (ESCMID) for helping distribute the questionnaires.

\section{Funding}

The HPV Prevention and Control Board is supported by in-kind contributions and support from the international experts involved and their institutions. To set up the activities and support publication cost, the secretariat obtains unrestricted grants from industry (GlaxoSmithKline Biologicals (GSK), MSD, Roche, and Hologic). All funds are handled according to the rules of the University of Antwerp. No remuneration for experts or speakers is provided.

\section{Conflict of interest}

MP is based in a laboratory that performs HPV and COVID-19 molecular testing. He declares no other conflict of interest.

$\mathrm{KC}$ is based in a laboratory that performs HPV testing, and KC's institution has received research funding or free-of-charge consumables to support research from the following commercial entities in the last 3 years: Cepheid, Euroimmun, GeneFirst, SelfScreen, Hiantis, Seegene, Roche, Abbott, and Hologic.

$\mathrm{AV}$ is based at the University of Antwerp, which obtained unrestricted educational grants from GSK, MSD, Roche, and Hologic; $\mathrm{AV}$ received speaker's fees from MSD, which were paid directly to an educational fund held by the University of Antwerp. DNW and MB declare no conflict of interest.

Roche Diagnostics, Abbott Laboratories, Cepheid, Becton Dickinson, and Hologic were invited to provide input.

\section{References}

1. Simms KT, Steinberg J, Caruana M, Smith MA, Lew JB, Soerjomataram I, et al. Impact of scaled up human papillomavirus vaccination and cervical screening and the potential for global elimination of cervical cancer in 181 countries, 2020-99: a modelling study. Lancet Oncol. 2019;20:394-407.

2. Brisson M, Kim JJ, Canfell K, Drolet M, Gingras G, Burger EA, et al. Impact of HPV vaccination and cervical screening on cervical cancer elimination: a comparative modelling analysis in 78 low-income and lower-middle-income countries. Lancet. 2020;395:575-90.

3. Canfell K, Kim JJ, Brisson M, Keane A, Simms KT, Caruana M, et al. Mortality impact of achieving WHO cervical cancer elimination targets: a comparative modelling analysis in 78 low-income and lower-middle-income countries. Lancet. 2020;395:591-603.

4. Poljak M, Oštrbenk Valenčak A, Gimpelj Domjanič G, Xu L, Arbyn M. Commercially available molecular tests for human papillomaviruses: a global overview. Clin Microbiol Infect. 2020;26:1144-50.

5. World Health Organization. In vitro diagnostics and laboratory technology [Internet]. 2020. Available from: https://www.who.int/diagnostics_laboratory/ evaluations/pq-list/public_report_hpv/en.

6. Center for Systems Science and Engineering at Johns Hopkins University. COVID-19 Dashboard [Internet]. 2021. Available from: https://gisanddata.maps.arcgis.com/apps/opsdashboard/index.html\#/bda759474ofd40299423467 b48egecf6.

7. World Health Organization. Rapid assessment of service delivery for noncommunicable diseases (NCDs) during the COVID-19 pandemic. Geneva; 2020.

8. Dinmohamed AG, Visser O, Verhoeven RHA, Louwman MWJ, van Nederveen FH, Willems SM, et al. Fewer cancer diagnoses during the COVID-19 epidemic in the Netherlands. Lancet Oncol. 2020;21:750-1.

9. Tsibulak I, Reiser E, Bogner G, Petru E, Hell-Teutsch J, Reinthaller A, et al. Decrease in gynecological cancer diagnoses during the COVID-19 pandemic: an Austrian perspective. Int J Gynecol Cancer. 2020;30:1667-71.

10. Skovlund CW, Friis S, Dehlendorff C, Nilbert MC, Mørch LS. Hidden morbidities: drop in cancer diagnoses during the COVID-19 pandemic in Denmark. Acta Oncol. 2021;60:20-3.

11. Jacob L, Loosen SH, Kalder M, Luedde T, Roderburg C, Kostev K. Impact of the COVID-19 pandemic on cancer diagnoses in general and specialized practices in Germany. Cancers (Basel). 2021;13:408.

12. Maluchnik M, Podwójcic K, Więckowska B. Decreasing access to cancer diagnosis and treatment during the COVID-19 pandemic in Poland. Acta Oncol. 2021;60:28-31.

13. Castanon A, Rebolj M, Pesola F, Sasieni P. Recovery strategies following COVID-19 disruption to cervical cancer screening and their impact on excess diagnoses. Brit J Cancer. 2021. Epub ahead of print.

14. Miller MJ, Xu L, Qin J, Hahn EE, Ngo-Metzger Q, Mittman B, et al. Impact of COVID-19 on cervical cancer screening rates among women aged 21-65 years in a large integrated health care system-southern California, January 1 - September 30, 2019, and January 1 - September 30, 2020. MMWR Morb Mortal Wkly Rep. 2021;70:109-13.

15. HPV Prevention and Control Board. Challenges in the HPV screening landscape, triage of screening positive samples, and screening in the era of vaccination [Internet]. 2020. Available from: https://www.uantwerpen.be/en/projects/hpvprevention-and-control-board/meetings/technical-meeting-august-2020. 
16. HPV Prevention and Control Board. Impact of COVID-19 on cervical cancer screening, treatment and vaccination [Internet]. 2020. Available from: https:// www.uantwerpen.be/en/projects/hpv-prevention-and-control-board/meetings/technical-meeting-november-2020.

17. Gao J, Wu L, Yang D, Gong W, Wang J. A one-pot CRISPR/Cas9-typing PCR for DNA detection and genotyping. J Mol Diagn. 2021;23:46-60.

18. Ramachandran A, Huyke DA, Sharma E, Sahoo MK, Huang C, Banaei N, et al. Elec tric field-driven microfluidics for rapid CRISPR-based diagnostics and its application to detection of SARS-CoV-2. Proc Natl Acad Sci U S A. 2020;117:29518-25.
19. Thompson D, Lei Y. Mini review: recent progress in RT-LAMP enabled COVID-19 detection. Sensors and Actuators Reports. 2020;2:100017.

20. Steben M, Norris T, Rosberger Z. COVID-19 won't be the last (or worst) pandemic: it's time to build resilience into our cervical cancer elimination goals. J Obstet Gynaecol Can. 2020;42:1195-6.

21. Ajenifuja KO, Belinson J, Goldstein A, Desai KT, de Sanjose S, Schiffman M. Designing low-cost, accurate cervical screening strategies that take into account COVID-19: a role for self-sampled HPV typing2. Infect Agent Cancer. 2020;15:61. 